\title{
A rare presentation of skull-base osteomyelitis with neurovascular sheath extension following external otitis resolved by PET/MRI
}

\author{
Nicolas Louarn ${ }^{1} \cdot$ Quentin Alias $^{2} \cdot$ Laurène Aupin $^{1} \cdot$ Nicolas Benoist $^{1} \cdot$ Marine Desroches $^{3} \cdot$ Alain Luciani $^{1}$. \\ Damien Bresson ${ }^{4}$. Jérôme Hodel ${ }^{1,2} \cdot$ Emmanuel $\mathrm{Itti}^{1}$
}

Received: 5 April 2018 / Accepted: 14 May 2018 / Published online: 24 May 2018

(C) Springer-Verlag GmbH Germany, part of Springer Nature 2018

\author{
Abbreviations \\ ${ }^{18}$ F-FDG ${ }^{18}$ F-Fluorodeoxyglucose \\ PET Positron emission tomography \\ MRI Magnetic resonance imaging \\ SUV Standardized uptake value
}

\begin{abstract}
${ }^{18}$ F-FDG PET/MRI was performed on a 67 -year-old diabetic man referred for staging of a possible tumour revealed by multiple cranial nerve impairment (VII, IX, X, XI and XII) and skull-base osteolysis on CT. The PET image (a) shows heterogeneous uptake in the occipital bone and surrounding soft tissues (full arrow, SUVmax 5.0), with extension around the right condyle (dotted arrow). The contrast-enhanced T1weighted MRI image (b) reveals hyperintensity in the clivus and the right occipital condyle/petrous apex, infiltrating contiguous soft tissues (full arrow), and focal posterior leptomeningeal enhancement (dotted arrow). The areas of hyperintensity coincide on the fused image (c) with the ${ }^{18} \mathrm{~F}$ FDG uptake, and are clearly seen on the diffusion-weighted
\end{abstract}

Nicolas Louarn

n.louarn@gmail.com

1 SyMPTOm PET/MRI platform, Nuclear Medicine Department, Henri Mondor University Hospital, AP-HP, 51 avenue du Maréchal de Lattre de Tassigny, 94010 Créteil, France

2 Neuroradiology Department, Henri Mondor University Hospital, AP-HP, F-94010 Créteil, France

3 Microbiology Department, Henri Mondor University Hospital, AP-HP, F-94010 Créteil, France

4 Neurosurgery Department, Henri Mondor University Hospital, AP-HP, F-94010 Créteil, France image (d). Linear ${ }^{18} \mathrm{~F}$-FDG uptake in the right neurovascular structures is also shown on the coronal slices (e-g) and maximum intensity projection (h, arrowhead) with jugular and carotid compression on MRI. The association between moderate, diffuse ${ }^{18}$ F-FDG uptake and infiltrative contrast enhancement suggested osteomyelitis. The medical history indicated that this infection probably complicated a right otitis externa which occurred several weeks before and was treated with broad-spectrum antibiotics. Signs of active otitis are still visible on the PET/MRI images that show mild ${ }^{18}$ F-FDG uptake (stars, SUVmax 2.0) and contrast-enhanced wall thickening of the external auditory canal. The diagnosis was confirmed by an endonasal endoscopy biopsy carried out a few days later, that showed Pseudomonas aeruginosa and Stenotrophomonas maltophilia infections, and allowed an adapted antibacterial therapy to be started. This case illustrates the complementary value of PET and MRI for characterizing cell metabolism and the tissue microenvironment in osteomyelitis of the vertebrae [1] and skull base [2,3] in a one-shot setting.

\section{References}

1. Kouijzer IJE, Scheper H, de Rooy JWJ, Bloem JL, Janssen MJR, van den Hoven L, et al. The diagnostic value of 18F-FDG-PET/CT and MRI in suspected vertebral osteomyelitis - a prospective study. Eur J Nucl Med Mol Imaging. 2018;45:798-805.

2. van Kroonenburgh AMJL, van der Meer WL, Bothof RJP, van Tilburg M, van Tongeren J, Postma AA. Advanced imaging techniques in skull base osteomyelitis due to malignant otitis externa. Curr Radiol Rep. 2018;6:3.

3. Nomura M, Shin M, Ohta M, Nukui Y, Ohkusu K, Saito N. Atypical osteomyelitis of the skull base and craniovertebral junction caused by Actinomyces infection - case report. Neurol Med Chir. 2011;51:64-6. 